\title{
The type III TGF- $\beta$ receptor suppresses breast cancer progression
}

\author{
Mei Dong, ${ }^{1}$ Tam How, ${ }^{1}$ Kellye C. Kirkbride, ${ }^{1,2}$ Kelly J. Gordon,1,2 Jason D. Lee,1,2 Nadine Hempel, ${ }^{1}$ \\ Patrick Kelly, ${ }^{2}$ Benjamin J. Moeller, ${ }^{3}$ Jeffrey R. Marks, ${ }^{4}$ and Gerard C. Blobe ${ }^{1,2}$
}

1Department of Medicine, 2Department of Pharmacology and Cancer Biology, ${ }^{3}$ Department of Radiation Oncology, and ${ }^{4}$ Department of Surgery, Duke University Medical Center, Durham, North Carolina, USA.

\begin{abstract}
The TGF- $\beta$ signaling pathway has a complex role in regulating mammary carcinogenesis. Here we demonstrate that the type III TGF- $\beta$ receptor (T $\beta$ RIII, or betaglycan), a ubiquitously expressed TGF- $\beta$ coreceptor, regulated breast cancer progression and metastasis. Most human breast cancers lost T $\beta$ RIII expression, with loss of heterozygosity of the TGFBR3 gene locus correlating with decreased T $\beta$ RIII expression. T $\beta$ RIII expression decreased during breast cancer progression, and low T $\beta$ RIII levels predicted decreased recurrence-free survival in breast cancer patients. Restoring T $\beta$ RIII expression in breast cancer cells dramatically inhibited tumor invasiveness in vitro and tumor invasion, angiogenesis, and metastasis in vivo. T $\beta$ RIII appeared to inhibit tumor invasion by undergoing ectodomain shedding and producing soluble T $\beta$ RIII, which binds and sequesters TGF- $\beta$ to decrease TGF- $\beta$ signaling and reduce breast cancer cell invasion and tumor-induced angiogenesis. Our results indicate that loss of T $\beta$ RIII through allelic imbalance is a frequent genetic event during human breast cancer development that increases metastatic potential.
\end{abstract}

\section{Introduction}

TGF- $\beta$ is a member of a superfamily of functionally diverse, but structurally conserved, cytokines that regulate cell proliferation, differentiation, apoptosis, and motility in a cell- and context-specific manner (1). TGF- $\beta$ exerts these biological effects by binding to 2 high-affinity cell surface receptors, the type II TGF- $\beta$ receptor (T $\beta$ RII) and the type III TGF- $\beta$ receptor (T $\beta$ RIII, or betaglycan); $T \beta R I I I$ functions as a coreceptor to increase ligand binding to T $\beta$ RII. Once bound to TGF- $\beta$, T $\beta$ RII recruits, binds, and transphosphorylates the type I TGF- $\beta$ receptor (T $\beta$ RI), thereby stimulating its protein kinase activity. The activated T $\beta \mathrm{RI}$ phosphorylates transcription factors Smad2 or Smad3, which then binds to Smad4. The resulting Smad complex translocates into the nucleus and interacts with other transcription factors to specifically regulate the transcription of a multitude of TGF- $\beta$-responsive genes.

TGF- $\beta$ has an important role in normal mammary biology as a potent inhibitor of mammary epithelial proliferation and regulator of mammary ductal and alveolar development $(2,3)$. Early in mammary carcinogenesis the TGF- $\beta$ signaling pathway functions as a tumor suppressor, with most human breast cancers developing resistance to the growth-inhibitory effects of TGF- $\beta$ and with elevated levels of TGF- $\beta$ associated with decreased incidence of mammary cancer in mouse models (4) and decreased breast cancer incidence in humans $(5,6)$. However, at later stages of mammary carcinogenesis, levels of TGF- $\beta$ increase with tumor progression (7-9) and confer a poorer prognosis for human breast cancer patients (10).

Although the TGF- $\beta$ signaling pathway has an important role in regulating mammary carcinogenesis, alterations in the main com-

Nonstandard abbreviations used: DCIS, ductal carcinoma in situ; ER, estrogen receptor; IDC, invasive ductal carcinoma; IHC, immunohistochemical; LOH, loss of heterozygosity; PCNA, proliferating cell nuclear antigen; sT $\beta$ RIII, soluble T $\beta$ RIII; T $\beta$ RI, type I TGF- $\beta$ receptor; T $\beta$ RII, type II TGF- $\beta$ receptor; T $\beta$ RIII, type III TGF- $\beta$ receptor; 4T1-Neo cells, 4T1 cells stably expressing the pcDNA-Neo expression vector; 4T1-T $\beta$ RIII cells, 4T1 cells stably expressing T $\beta$ RIII.

Conflict of interest: The authors have declared that no conflict of interest exists. Citation for this article: J. Clin. Invest. 117:206-217 (2007). doi:10.1172/JCI29293. ponents of the pathway, including T $\beta$ RII, T $\beta$ RI, Smad2, Smad3, and $S m a d 4$, are infrequent in human breast cancers $(6,11)$. A role for the TGF- $\beta$ coreceptor T $\beta$ RIII as a mediator and regulator of TGF- $\beta$ signaling has emerged as a result of recent studies, with essential roles in chick heart and mouse development $(12,13)$ and in regulating T $\beta$ RII and T $\beta$ RI cell surface expression and internalization as well as TGF- $\beta$ signaling $(14,15)$. T $\beta$ RIII has been reported to be expressed at low levels in the MCF-7 human breast cancer cell line (16), and restoring T $\beta$ RIII expression in these cells suppresses their anchorage-independent growth in vitro as assayed by colony formation in soft agarose (16), while increasing T $\beta$ RIII expression in MDA-MB231 breast cancer cells suppresses their tumorigenicity in vivo as assessed by tumor formation in athymic nude mice (17). These results suggest that decreased T $\beta$ RIII expression may be a mechanism for altering TGF- $\beta$ responsiveness during mammary carcinogenesis. Here we demonstrate that T $\beta$ RIII is a suppressor of breast cancer progression and that, when T $\beta$ RIII expression is restored in human breast cancer cells, breast tumor invasion, angiogenesis, and metastasis are inhibited in vivo.

\section{Results}

Decreased T $\beta$ RIII expression in human breast cancer. As evidence supporting roles for T $\beta$ RIII in regulating TGF- $\beta$ signaling have emerged $(12-15)$, and a low level of T $\beta$ RIII expression has been reported in the MCF-7 human breast cancer cell line (16), we investigated the expression status of T $\beta$ RIII in human breast cancer. Breast cancers are classified into different histologic subtypes, with invasive ductal carcinoma (IDC) being the most common ( $70 \%)$, followed by lobular carcinoma ( $\sim 8 \%)$. The development of IDC has been proposed to follow a stepwise process - including ductal carcinoma in situ (DCIS) - culminating in the potentially lethal stage of IDC. We initially analyzed a cDNA array containing 50 human breast cancer samples with matched normal controls (Figure 1A). T $\beta$ RIII mRNA levels were reduced in $60 \%$ of the lymph node-negative IDCs (2.64 \pm 0.49 -fold), $64.7 \%$ of the lymph node-positive IDCs $(2.47 \pm 0.29$ fold) and in $100 \%$ of the IDCs with distant metastasis (3.98 \pm 0.79 
A

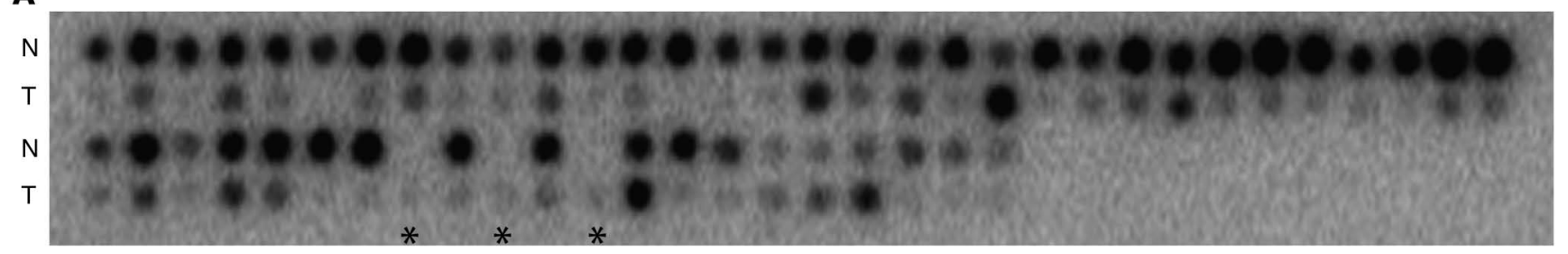

\begin{tabular}{|c|c|c|c|c|c|c|c|}
\hline & DCIS & IDC & $\begin{array}{c}\text { Lobular } \\
\text { carcinoma }\end{array}$ & $\begin{array}{c}\text { Tubular } \\
\text { carcinoma }\end{array}$ & $\begin{array}{c}\text { Medullary } \\
\text { carcinoma }\end{array}$ & $\begin{array}{c}\text { Mucinous } \\
\text { carcinoma }\end{array}$ & Fibrosarcoma \\
\hline No. of samples & 1 & 38 & 6 & 2 & 1 & 1 & 1 \\
\hline Ratio (N/T) & 2.94 & $3.12 \pm 0.22$ & $2.84 \pm 0.40$ & $2.28 \pm 0.16$ & 0.69 & 2.75 & 2.52 \\
\hline
\end{tabular}

C

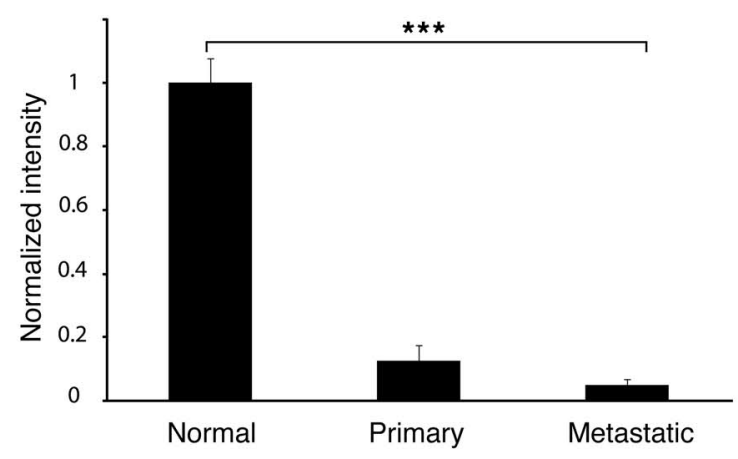

Figure 1

Loss of T $\beta$ RIII mRNA expression during mammary carcinogenesis. (A) T $\beta$ RIII mRNA levels were detected by hybridizing [32P]-labeled human T $\beta$ RIII cDNA probe to the Clontech Cancer Profiling Array I. The portion of the array containing breast samples is shown, with tumor specimens $(\mathrm{T})$ and matched normal breast tissue $(\mathrm{N})$. Asterisks indicate metastatic specimens corresponding to the normal and tumor samples spotted on the immediate left. (B) Quantitative data were obtained by analyzing the array with NIH ImageJ software, summarized as the ratio relative to normal breast, and expressed as mean \pm SEM. (C) Quantitative data from matched normal, primary breast tumor, and metastatic breast tumor tissue expressed as mean \pm SEM. ${ }^{* * \star} P<0.0001$, ANOVA.

fold) as well as in all histological subtypes represented in Figure 1B, suggesting an increased frequency of loss with disease progression. T $\beta$ RIII mRNA levels were also significantly reduced in $83.3 \%$ of lobular carcinomas ( $2.84 \pm 0.40$-fold). We also examined 3 sets of specimens on the cDNA array with matched normal breast, primary breast cancer, and metastatic breast cancer tissue from the same patient. In all 3 cases, T $\beta$ RIII expression decreased from normal breast to primary breast cancer to metastatic breast cancer, with an average $88 \%$ decrease in expression from normal breast to primary breast cancer and a further $61 \%$ decrease from primary breast cancer to metastatic breast cancer (Figure 1C; $P<0.0001$ ), suggesting progressive loss of T $\beta$ RIII expression with cancer progression.

To confirm decreased expression of T $\beta$ RIII and establish its association with breast cancer progression, we performed immunohistochemical (IHC) analysis for T $\beta$ RIII expression on a breast cancer tissue array containing 252 breast cancers of different stages (20 DCIS, 64 lymph node-negative, 64 lymph node-positive, and 64 distant metastatic) and 40 normal breast specimens with available pathologic information including tumor size, TNM stage, number of nodes positive, invasive grade, and estrogen receptor (ER) and progesterone receptor status. T $\beta$ RIII expression progressively decreased from normal breast specimens (Figure 2, A and B) to DCIS to lymph node-negative breast cancer. The proportion with abundant T $\beta$ RIII expression decreased from $68.4 \%$ in normal breast specimens to $23.5 \%$ in DCIS specimens to $5.1 \%$ in lymph node-nega- tive breast cancer specimens $(P<0.01,2$-tailed Fisher's exact probability). At the same time, the proportion with no T $\beta$ RIII expression increased from $0 \%$ in normal breast specimens to $23.5 \%$ in DCIS specimens to $67.8 \%$ in lymph node-negative breast cancer specimens $(P<0.01,2$-tailed Fisher's exact probability). In DCIS specimens with loss of T $\beta$ RIII expression (Figure $2 \mathrm{~A}$, arrow), T $\beta$ RIII was present in adjacent normal-appearing breast ducts (Figure 2A, arrowhead), which served as a useful internal control. To directly address the role of loss of T $\beta$ RIII expression in breast cancer progression, we assessed matched tissue sets for which either matching normal breast and invasive breast cancer specimens (Figure 2C) or matching DCIS and invasive breast cancer specimens (Figure 2D) were available for analysis. In addition, one of these samples had matching normal breast, DCIS, and invasive breast cancer specimens available for analysis. When examining T $\beta$ RIII expression in matched normal breast and invasive breast cancer specimens, T $\beta$ RIII expression decreased in every case (10 of 10), with 6 cases decreasing from high expression (IHC score of 5) in normal breast tissue to low expression (IHC score of $0-1$ ) in the matching invasive breast cancer tissue (Figure 2C). When examining T $\beta$ RIII expression in matched DCIS and invasive breast cancer specimens, T $\beta$ RIII expression decreased in $63 \%$ of the cases ( 5 of 8 ), with 1 additional case where expression was already absent at the DCIS stage (Figure 2D). In the sample with matching normal breast, DCIS, and invasive breast cancer specimens, T $\beta$ RIII expression decreased from an IHC score of 5 in the 

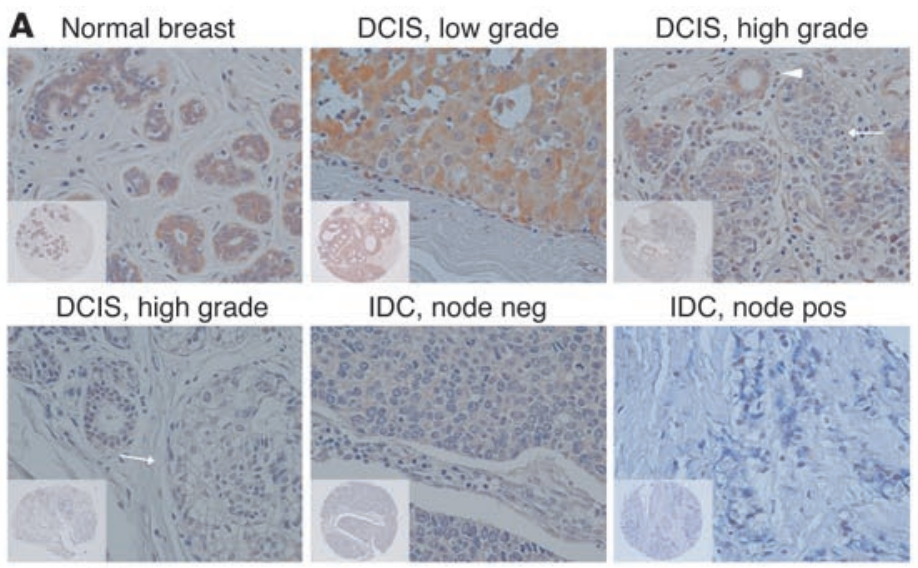

IDC, node neg

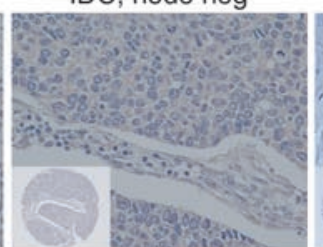

IDC, node pos

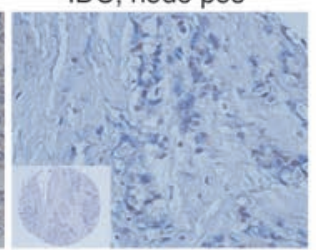

C

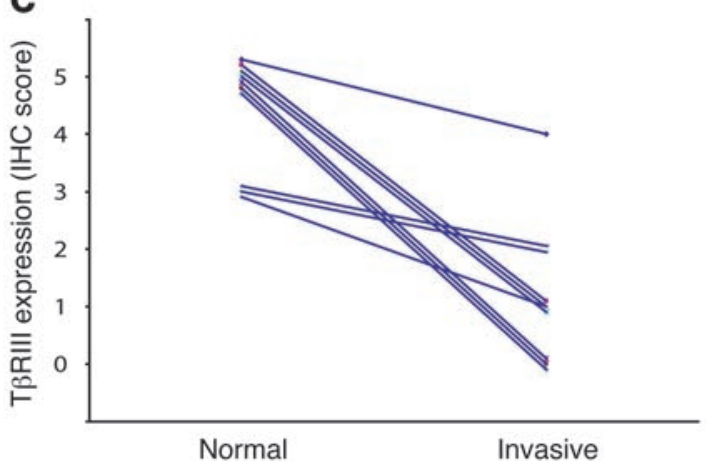

B

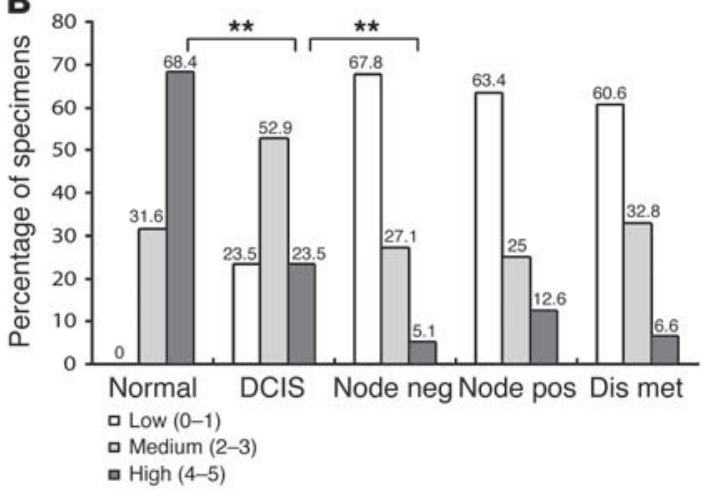

D

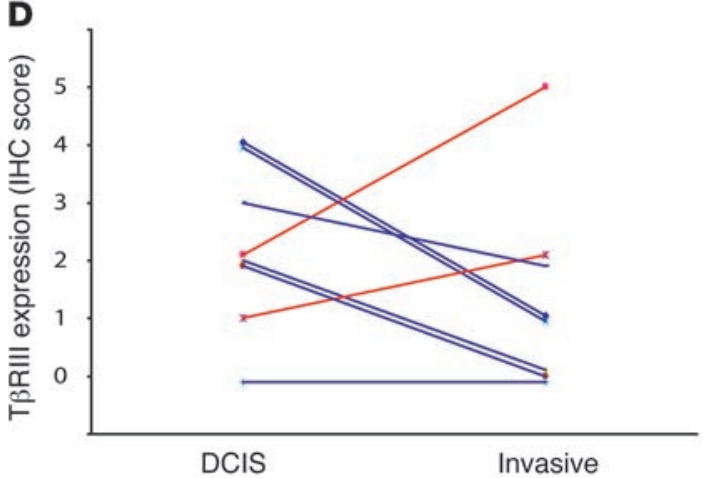

Figure 2

Progressive loss of T $\beta$ RIII protein expression during mammary carcinogenesis. (A) Representative IHC analysis of T $\beta$ RIII expression (original magnification, $\times 40$ ) in normal breast ductal cells, in different grades of DCIS, and in lymph node-negative (node neg) and -positive (node pos) IDC. Insets depict staining of entire tissue core (original magnification, $\times 10$ ). Immunoreactivity for T $\beta$ RIII was scored as $0-5$ and categorized as low (0-1), medium (2-3), or high (4-5). Note the absence of T $\beta$ RIII staining in IDC and high-grade DCIS (arrows) versus presence of staining in normal ducts and normal-appearing ducts adjacent to the DCIS lesion (arrowhead). (B) Summary of IHC results, with percentages shown. Dis met, distant metastasis. ${ }^{*} P<0.01$, 2-tailed Fisher's exact probability. (C) Patient-matched normal and invasive breast cancer IHC T $\beta$ RIII scores. (D) Patient-matched DCIS and invasive breast cancer IHC T $\beta$ RIII scores.

normal breast specimen to 2 in the DCIS specimen to 0 in the invasive breast cancer specimen. These data indicate that T $\beta$ RIII expression is significantly decreased in breast cancer, with loss of T $\beta$ RIII expression correlating with breast cancer progression.

Loss of heterozygosity and transcriptional downregulation of the T $\beta R I I I$ gene in buman breast cancer. Members of the TGF- $\beta$ signaling pathway, including T $\beta$ RII and Smad4, frequently have inactivating mutations in human cancers $(18,19)$. To investigate whether there are mutations in the T $\beta$ RIII gene, TGFBR3 (216 kb of genomic DNA composed of 17 exons), that could abrogate T $\beta$ RIII function in breast cancer, sequence analysis of the 16 coding exons (exon 1 is untranslated) was carried out on 20 primary breast cancer DNA samples. Although several polymorphisms were detected (data not shown), no mutations were found. Thus, TGFBR3 does not appear to be a target for mutational inactivation in breast cancer.

TGFBR3 maps to chromosome $1 \mathrm{p} 32$, a region that has been reported to exhibit loss of heterozygosity $(\mathrm{LOH})$ in a variety of human cancers, including breast cancer (20-22). Therefore, to investigate the mechanism for loss of T $\beta$ RIII expression during breast tumorigenesis, we examined $\mathrm{LOH}$ at the TGFBR3 locus using microsatellite markers on DNA samples extracted from 26 human breast cancer specimens and the matching normal peripheral lymphocytes. With 4 microsatellite markers immediately adjacent to and within the
TGFBR3 locus, we were able to establish that $50 \%$ (13 of 26) of these samples exhibited $\mathrm{LOH}$ at the TGFBR3 locus (Figure 3, A and B), closely matching the $43 \%-61 \% \mathrm{LOH}$ reported for the $1 \mathrm{p}$ region and the $58 \%$ reported for $1 \mathrm{p} 32$ in human breast cancers $(20-22)$. LOH at the TGFBR3 locus correlated with loss of T $\beta$ RIII expression, with $75 \%$ (9 of 12) of those with the lowest T $\beta$ RIII expression exhibiting LOH at the TGFBR3 locus and only $20 \%$ (1 of 5) with the highest T $\beta$ RIII expression exhibiting LOH at the TGFBR3 locus (Figure 3C). Taken together, these data support LOH as a mechanism for loss of T $\beta$ RIII expression in breast cancer.

During later stages of mammary carcinogenesis, levels of TGF- $\beta$ increase with tumor progression (7-9) and confer a poorer prognosis for human breast cancer patients (10). As TGF- $\beta$ isoforms have previously been demonstrated to decrease T $\beta$ RIII promoter activity (23), we assessed whether the elevated levels of TGF- $\beta$ could repress $\mathrm{T} \beta \mathrm{RIII}$ expression at the transcriptional level in breast cancer cells. In MDA-MB231 breast cancer cells, which exhibit basal T $\beta$ RIII expression, TGF- $\beta 1$ treatment resulted in a significant (up to $80 \%$ ) reduction in the T $\beta$ RIII mRNA level (Figure 3D). This effect was relatively specific for T $\beta$ RIII, as TGF- $\beta 1$ treatment slightly increased $T \beta R I$ mRNA levels and decreased T $\beta$ RII mRNA levels by less than $50 \%$ (Figure $3 \mathrm{D}$ ). These results suggest that, apart from $\mathrm{LOH}$, transcriptional downregulation due to increased TGF- $\beta$ in the breast 
A

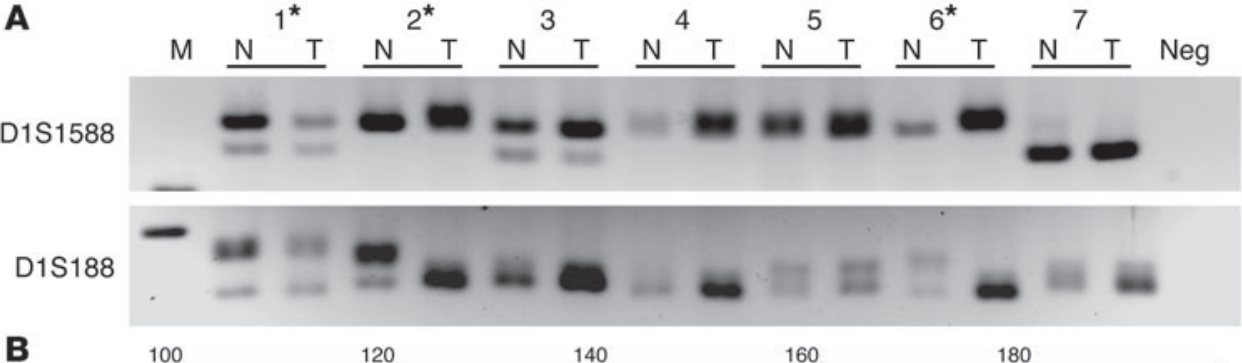

B
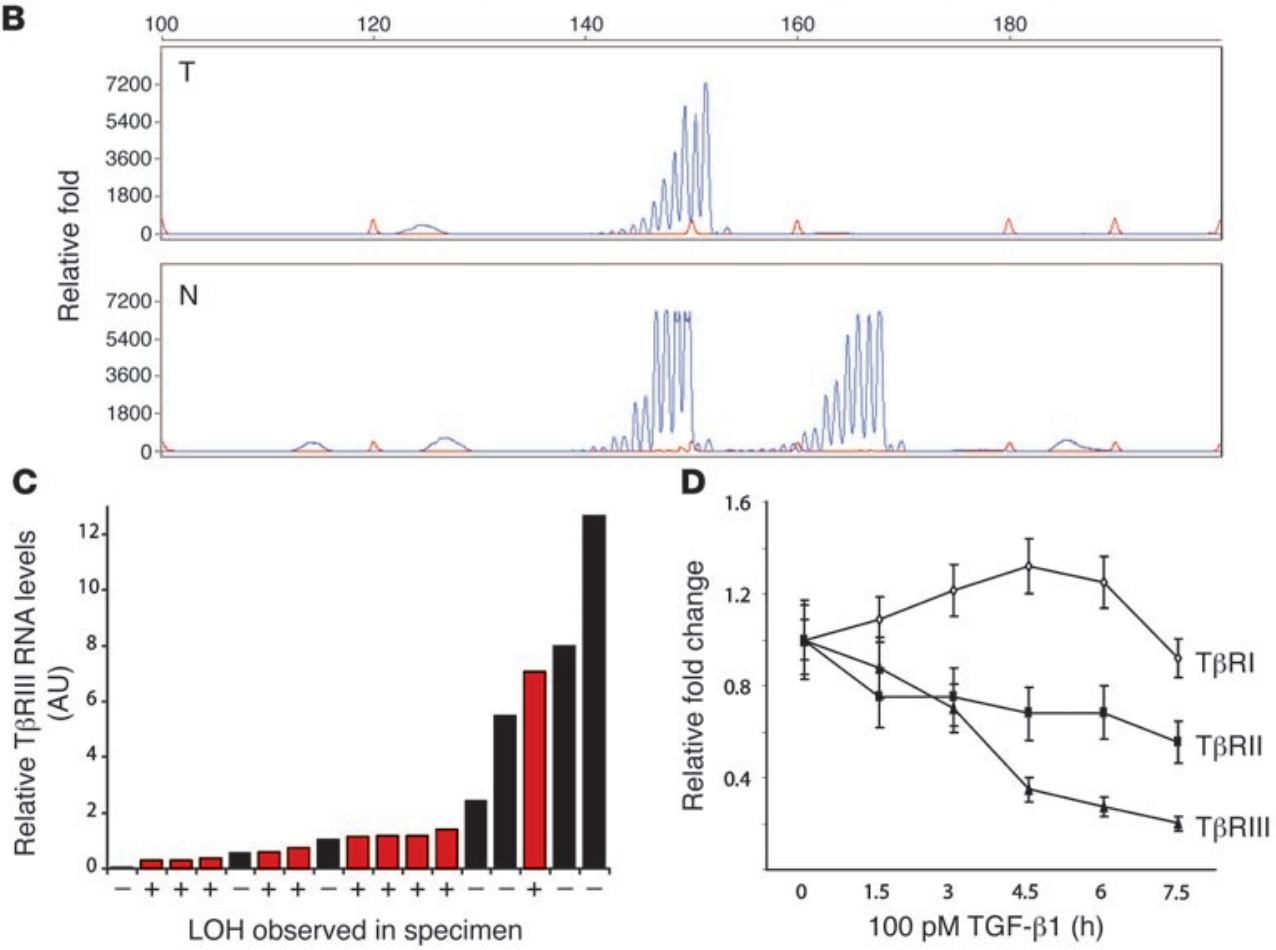

\section{Figure 3}

Frequent $\mathrm{LOH}$ of the TGFBR3 gene locus in human breast cancers correlates with loss of T $\beta$ RIII mRNA expression. $\mathrm{LOH}$ analysis was performed on DNA extracted from 26 human breast cancer specimens and matching normal lymphocytes. (A) Representative results showing allelic loss in tumors 1, 2, and 6 (denoted by asterisks) when PCR products were separated on a MetaPhor agarose gel. Microsatellite markers D1S1588 and D1S188 are described in Methods. (B) $\mathrm{LOH}$ was confirmed using an $\mathrm{ABI}$ sequencer and quantified using GeneScan software. A representative sample with $\mathrm{LOH}$ is shown. (C) Quantitative real-time PCR analysis of T $\beta R I I I$ mRNA levels in breast cancer specimens with (red bars) and without (black bars) LOH. (D) Quantitative realtime PCR analysis of mRNA levels of $T \beta R I, T \beta R I I$, and $T \beta R I I I$ in MDA-MB231 cells in response to TGF- $\beta 1$ (100 pM) stimulation for the indicated times. cancer microenvironment could be another mechanism leading to decreased T $\beta$ RIII expression during mammary carcinogenesis.

$T \beta R I I I$ delays and decreases metastatic potential of breast cancer cells in vivo. The frequent loss of T $\beta$ RIII expression observed during progression to invasive disease suggested that T $\beta$ RIII loss during mammary carcinogenesis may specifically promote tumor invasion and metastasis in vivo. To investigate a causal role for decreased T $\beta$ RIII expression in breast cancer progression, we examined the effect of T $\beta$ RIII on in vivo tumor growth and metastasis using a murine model for mammary carcinogenesis. Murine 4T1 mammary cancer cells, which are derived from a BALB/c murine mammary tumor, share many characteristics with human mammary cancers including spontaneous lung metastasis in immunocompetent mice and have been widely used as a model of breast cancer $(24,25)$. The $4 \mathrm{~T} 1$ cells were genetically engineered to express the firefly luciferase gene so that by periodically injecting the substrate luciferin into mice carrying these cells and taking bioluminescent images, we were able to closely and quantitatively follow their in vivo growth and metastatic potential. The 4T1 cells were stably transfected with T $\beta$ RIII (4T1-T $\beta$ RIII cells, see Supplemental Figure 1; supplemental material available online with this article; doi:10.1172/JCI29293DS1), resulting in 4T1 cells with increased T $\beta$ RIII expression. The 4T1-T $\beta$ RIII cells and control 4T1 cells stably expressing the pcDNA-Neo expression vector (4T1-Neo cells) were injected into the axillary mammary fat pads of BALB/c mice. The primary tumor was measured every 2 days starting from day 10 after injection and removed on day 20. Tumor metastases were then followed by bioluminescent imaging every 3 days over a period of 19 days. No significant difference was observed in the growth of the primary tumors from 4T1-T $\beta$ RIII and 4T1-Neo cells as shown by the growth curve (Figure $4 \mathrm{~A}$ ) and tumor mass at the time of resection (Figure 4B), establishing that T $\beta$ RIII had no effect on tumorigenicity in vivo. However, mice injected with 4T1-T $\beta$ RIII cells demonstrated a significantly delayed onset of tumor metastasis as well as a significant reduction in both the size and number of lung metastases compared with the mice injected with control 4T1-Neo cells (Figure 4, $\mathrm{C}-\mathrm{E})$. In addition, while no tumor recurrence at the primary site or animal death was observed in mice injected with 4T1-T $\beta$ RIII cells, the control mice with the 4T1-Neo cells had a $20 \%$ local recurrence rate and a $13.3 \%$ death rate during the study (Table 1 ).

Further pathologic examination of the primary tumors demonstrated that the 4T1-Neo tumors exhibited increased invasion of the surrounding normal mammary tissue (Figure 5A) and skin (Figure 5B), while the 4T1-T $\beta$ RIII tumors exhibited little to no invasion and instead maintained a distinct margin with the adjacent normal tissue (Figure 5C). In addition, primary recurrences in the 4T1-Neo mice exhibited invasion of tumor cells into the blood vessels, resulting in internal hemorrhage (Figure 5D). Pathologic examination of tumor metastasis revealed distant metastasis to the 
A

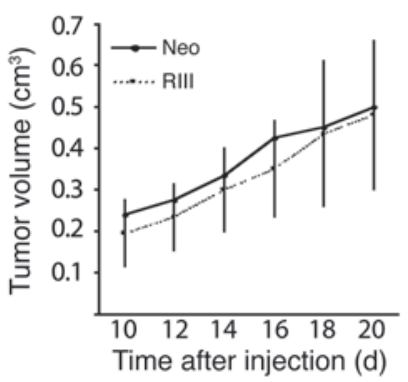

C

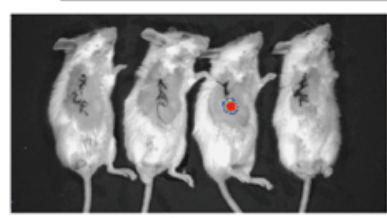

POD4

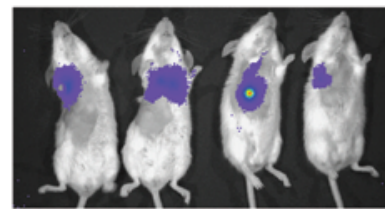

POD10

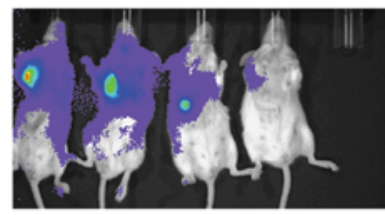

POD16
B

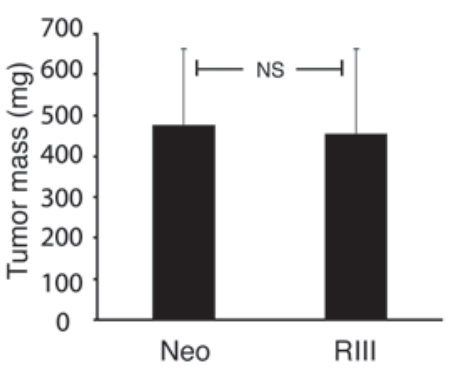

$\mathrm{Neo}$

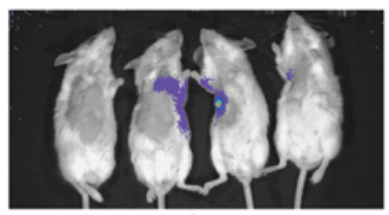

POD7

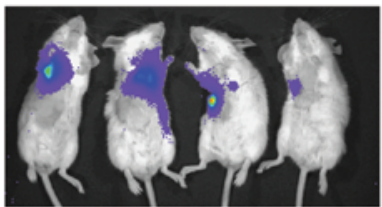

POD13

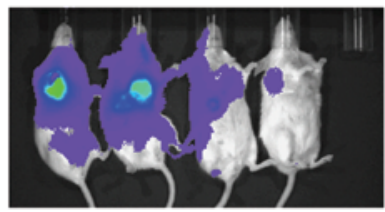

POD19

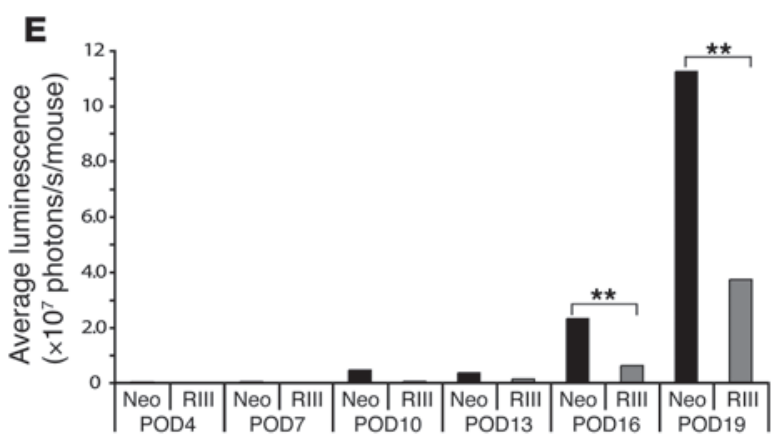

RIII

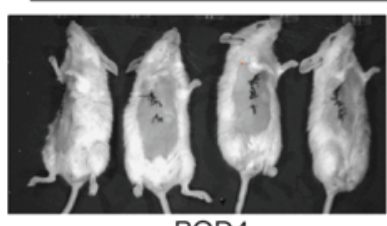

POD4

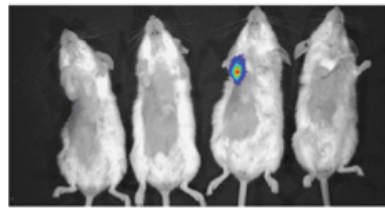

POD10

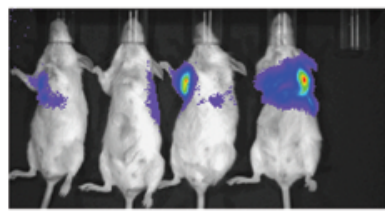

POD16

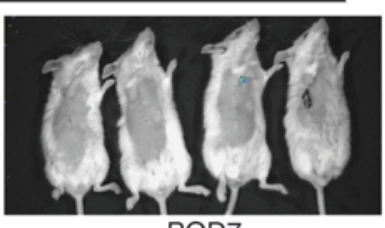

POD7

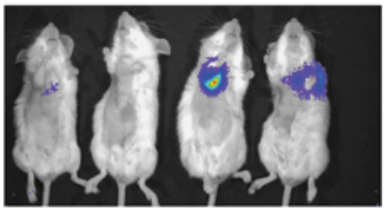

POD13

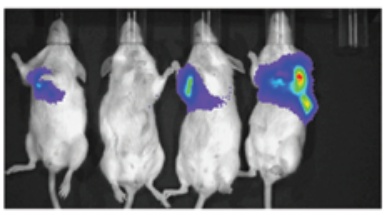

POD19

D

\begin{tabular}{|c|c|c|c|c|c|c|}
\hline & \multicolumn{5}{|c|}{ Luminescent signal intensity (photons/s) } \\
\hline & & No signal & $10^{5} \longrightarrow$ & $10^{6} \longrightarrow$ & ${ }^{7} \longrightarrow$ & $10^{8} \longrightarrow 10^{9}$ \\
\hline \multirow{2}{*}{ POD4 } & \multirow{2}{*}{$\begin{array}{l}\text { Neo } \\
\text { RIII }\end{array}$} & ............... & & - & & \\
\hline & & $* * * * * * * * * * * *$ & & & & \\
\hline \multirow{2}{*}{ POD7 } & Neo & $\cdots \cdots \cdots \cdot \cdots$ & •.・ & - & & \\
\hline & RIII & $* * * * * * * * * * * * *$ & & & & \\
\hline \multirow{2}{*}{ POD10 } & Neo & $\cdots . . .$. & & $\cdots \cdots$ & .• & \\
\hline & RIII & $* * * * * * * * * *$ & * & **** & & \\
\hline \multirow{2}{*}{ POD13 } & Neo & $\ldots \ldots \ldots$ & - & - & ... & \\
\hline & RIII & $* * * * * * *$ & $* * *$ & $* * * *$ & & \\
\hline \multirow{2}{*}{ POD16 } & Neo & $\ldots$ & .. $\cdot$ & .. & .. & 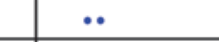 \\
\hline & RIII & $* * * * * * *$ & & $* * * * *$ & * & \\
\hline \multirow{2}{*}{ POD19 } & Neo & $\ldots$ & $\ddot{*}$ & .• $\quad$ • & ... & .• \\
\hline & RIII & $* * * * * *$ & * & * & $* * * * *$ & \\
\hline
\end{tabular}

Figure 4

T $\beta$ RIII delayed and decreased metastatic potential of breast cancer cells in vivo. Either 4T1-Neo (Neo) or 4T1-T $\beta$ RIII (RIII) cells (75,000 cells/ mouse) were implanted into the axillary mammary fat pads of BALB/c mice. (A) Primary tumor growth was recorded by measuring tumor size every 2 days beginning at 10 days after injection and presented as mean \pm SEM. (B) Weight of the primary tumors upon surgical removal on day 20 after injection. Data are mean \pm SEM $(n=16)$. (C) Bioluminescence imaging was performed every 3 postoperative days (POD). Representative images are shown. Red and violet signals correspond to the maximum and minimum intensity values, respectively, with other colors representing the values in between. (D) Record of luminescent signals for every mouse in each group at the indicated time points. (E) Average luminescent signal in each group at the indicated time points. ${ }^{* *} P<0.01$.

mesentery (Figure 5E), the paratracheal lymph nodes (Figure 5F), and the cecum in addition to the lung in control 4T1-Neo mice, while 4T1-T $\beta$ RIII exhibited only lung metastases. In addition, when lung metastases were observed in 4T1-T $\beta$ RIII mice, these 
Table 1

T $\beta$ RIII decreases metastasis in vivo

\begin{tabular}{lcc} 
& 4T1-Neo & 4T1-T $\beta$ RIII \\
Local recurrence rate & $20 \%(3$ of 15$)$ & $0 \%$ \\
Metastasis onset & POD4 & POD10 \\
Distant metastatic sites & Lung, cecum, mesentery & Lung \\
Metastatic rate & $80 \%(12$ of 15$)$ & $53.3 \%(8$ of 15$)$ \\
Average tumor load & $1.12 \times 10^{8}$ & $3.7 \times 10^{7}$ \\
on POD19 (photons/s) & & \\
Death rate by P0D19 & $13.3 \%(2$ of 15$)$ & $0 \%$ \\
\hline
\end{tabular}

POD, postoperative day.

metastatic lesions were always small, well circumscribed, and isolated (Figure 5, H and I) compared with the large, locally invasive lung metastases observed in 4T1-Neo mice (Figure 5G). These studies support a specific suppressor effect of T $\beta$ RIII on cellular invasiveness and metastasis, but not on primary tumorigenesis.

$T \beta R I I I$ decreases angiogenesis in vivo. Cancer metastasis is a multistep process requiring the cells growing at the primary site to invade through the basement membrane, enter lymph or blood vessels, extravasate from the vessel, and then grow at the distant site. Many of the processes involved in primary tumorigenesis and growth of metastases are similar, including increased proliferation, decreased apoptosis, and increased angiogenesis. To further establish the mechanism of T $\beta$ RIII on decreasing metastasis in vivo, we performed immunohistochemistry for the proliferation marker proliferating cell nuclear antigen (PCNA), TUNEL staining as a marker for apoptosis, and immunohistochemistry for CD31 as an endothelial surface marker on primary tumors and metastatic lesions. There were no significant differences observed in PCNA or TUNEL staining in 4T1-Neo and 4T1T $\beta$ RIII primary tumors or lung metastases (Figure $6 \mathrm{~A}$ ), suggesting that differences in proliferation or apoptosis did not account for the differential metastatic behavior of 4T1-Neo and 4T1-T $\beta$ RIII cells. However, CD31 staining revealed a decrease in the number of tumor-associated blood vessels per field, smaller vessel diameters, and less staining intensity in 4T1-T $\beta$ RIII tumors (Figure 6B), which supported an inhibitory effect of T $\beta$ RIII on tumor angiogenesis. Taken together, these data indicate that loss of T $\beta$ RIII expression facilitates tumor metastasis in vivo not only through an increase in tumor cell invasiveness but also through enhanced tumor angiogenesis.

$T \beta R I I I$ inhibits the invasiveness of breast cancer cells through the generation of soluble T $\beta R I I I$. To further define the mechanisms by which T $\beta$ RIII regulated breast cancer invasiveness and metastasis in vivo, we examined the effect of increasing $\mathrm{T} \beta \mathrm{RIII}$ expression on the invasiveness of breast cancer cell lines in vitro. We initially assessed the 4T1-Neo and 4T1T $\beta$ RIII cell lines; however, these cell lines both tended to aggregate and were not significantly invasive in vitro (data not shown). Therefore, we used the tumorigenic, invasive, and metastatic MDA-MB231 cell line. Overexpression of T $\beta$ RIII had no significant effect on the rate of cell division, nor did it restore cell responsiveness to TGF- $\beta$-induced growth inhibition (Supplemental Figure 2). However, it dramatically repressed the ability of MDA-MB231 cells to invade through Matrigel and significantly attenuated the responsiveness of the MDA-MB231 cells to TGF- $\beta$-induced invasion (Figure 7, A-C). These results confirm a direct effect of T $\beta$ RIII on inhibiting breast cancer cell invasiveness.

We next assessed the ability of specific T $\beta$ RIII mutants to mediate this function. Interestingly, a T $\beta$ RIII mutant lacking the entire cytoplasmic domain inhibited breast cancer cell invasiveness to an extent similar to that of full-length T $\beta$ RIII (Figure 7, A-C), suggesting that the effect of T $\beta$ RIII on regulating invasion is independent of functions mediated by the cytoplasmic domain of T $\beta$ RIII, including binding $\mathrm{G} \alpha$-interacting protein-interacting protein, $\mathrm{C}$ terminus (GIPC) (26) and $\beta$-arrestin 2 (15) and mediating TGF- $\beta$ signaling (14).

The extracellular domain of T $\beta$ RIII can be proteolytically cleaved in the juxtamembrane region (27), and the resulting soluble T $\beta$ RIII (sTßRIII) has been demonstrated to suppress tumor growth and angiogenesis, potentially through binding and sequestering TGF- $\beta$ and preventing signaling through the membrane-bound receptors (28). To assess whether the effects of T $\beta$ RIII could be mediated by the production of sT $\beta$ RIII, we first examined whether the 4T1T $\beta$ RIII and MDA-MB231-T $\beta$ RIII cells lines produced sT $\beta$ RIII. We collected conditioned media from each cell line, crosslinked iodinated TGF- $\beta 1$, and specifically immunoprecipitated sT $\beta$ RIII with an antibody to the extracellular domain. These studies confirmed that both the 4T1-T $\beta$ RIII and the MDA-MB231-T $\beta$ RIII cell lines produced a significant amount of sT $\beta$ RIII (Figure 7F).
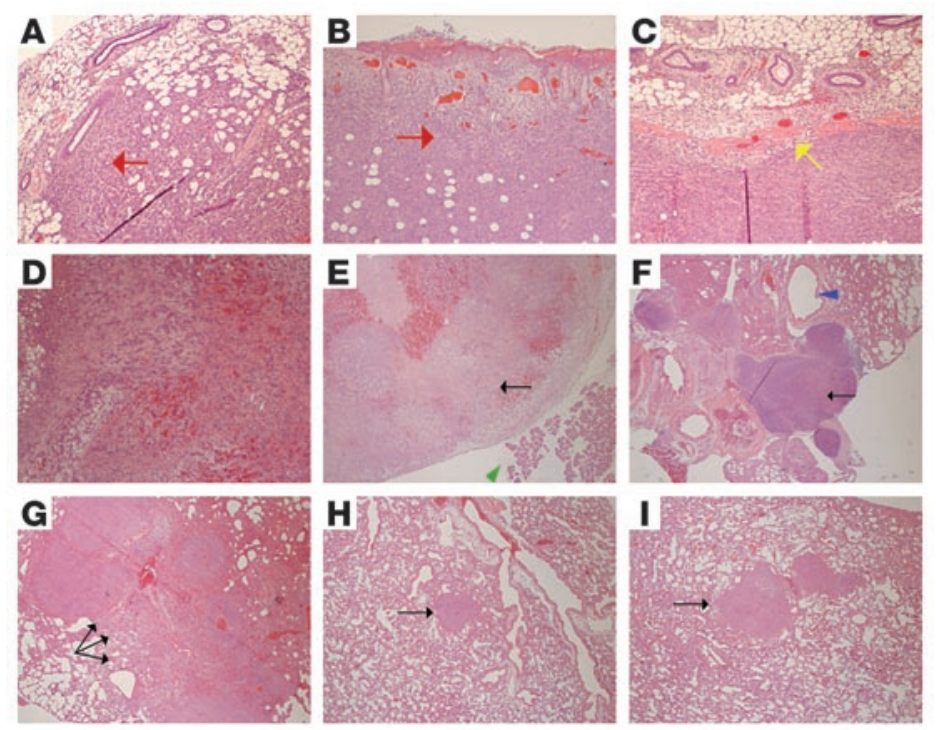

\section{Figure 5}

$T \beta R I I I$ decreased tumor cell invasiveness and metastasis in vivo. Representative H\&E staining (original magnification, $\times 10$ ) of (A and $\mathbf{B}$ ) primary tumors from mice implanted with 4T1-Neo cells exhibiting local invasion (red arrows) of tumor cells into the adjacent normal mammary tissue (A) and skin (B); (C) a representative primary tumor from mice implanted with 4T1-T $\beta$ RIII cells demonstrating the absence of local invasion, as indicated by the clear margin between the tumor and the adjacent normal mammary tissue (yellow arrow); (D) a recurring tumor in a mouse at the primary injection site of 4T1Neo cells exhibiting internal bleeding due to invasion of tumor cells into the blood vessels; (E) a metastatic tumor (black arrow) adjacent to the pancreas (green arrowhead) found on the mesentery of a mouse implanted with 4T1Neo cells; (F) a significantly enlarged paratracheal lymph node adjacent to the trachea (blue arrowhead) containing metastatic tumor cells (black arrow) in a mouse with 4T1-Neo cells, indicating the presence of lymphatic metastasis; (G) multiple large metastatic tumor nodules (black arrows) in the lung of a mouse implanted with 4T1-Neo cells; and ( $\mathbf{H}$ and $\mathbf{I})$ representative lung metastases in mice implanted with 4T1-T $\beta$ RIII cells (black arrows). 
A
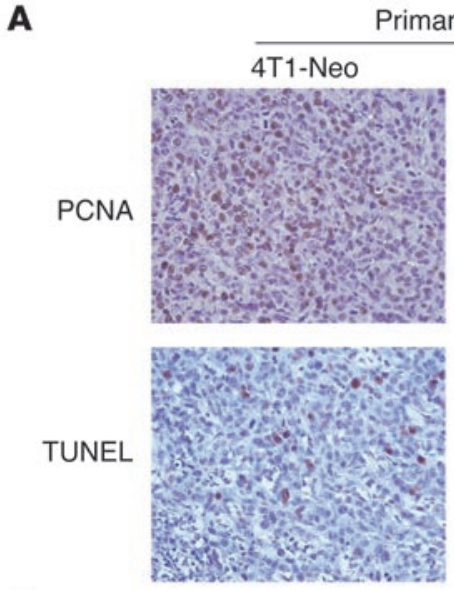

B

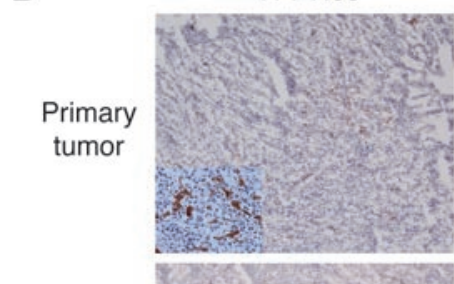

Lung metastasis

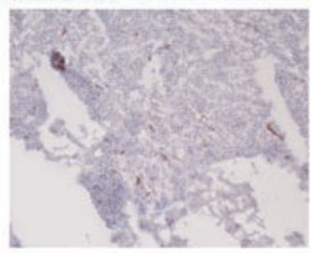

Primary tumor
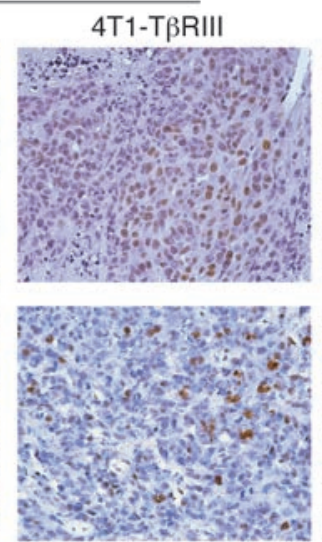

4T1-T $\beta$ RIII

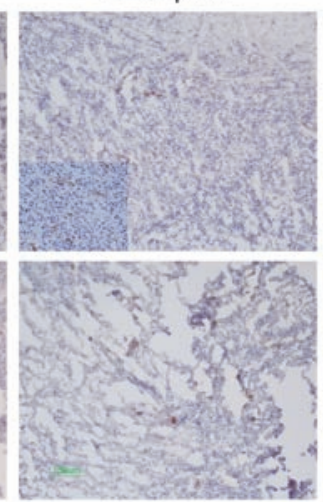

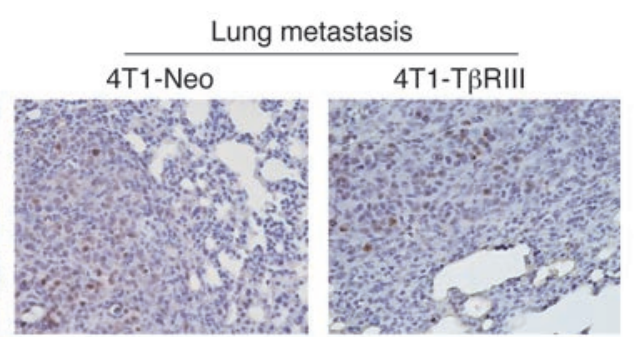
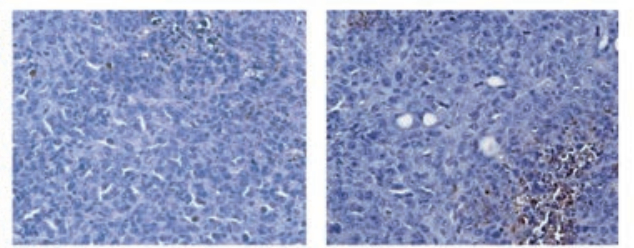

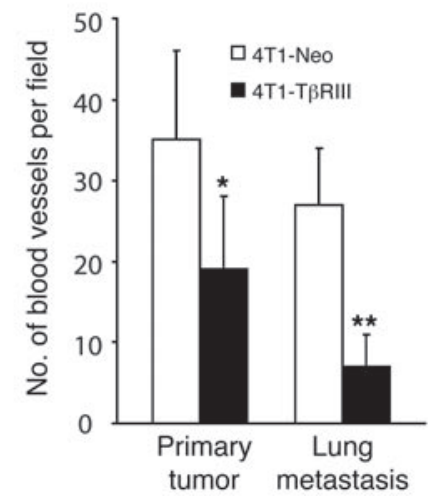

\section{Figure 6}

$\mathrm{T} \beta \mathrm{R} I \mathrm{II}$ inhibits tumor angiogenesis without altering cancer cell proliferation and apoptosis in vivo. (A) Tissue sections of primary tumors and lung metastases from mice implanted with 4T1-Neo and 4T1-T $\beta$ RIII cells were immunostained for PCNA and TUNEL to evaluate cell proliferation and apoptosis, respectively. Representative staining frequency and intensity is shown (original magnification, $\times 40$ ). (B) Immunostaining of CD31 (original magnification, $\times 10$ ) was performed as a marker to evaluate angiogenesis. Note the decreased number and size of tumor-associated blood vessels as well as decreased staining intensity (insets; original magnification, $\times 100$ ) in 4T1-T $\beta$ RIII primary tumors and lung metastases. Values are the averages from 6 mice and expressed as mean \pm SD. ${ }^{\star} P<0.05 ;{ }^{\star \star} P<0.01$.

Accordingly, we examined the effect of sTRRIII on MDA-MB231 breast cancer cell invasion in vitro. Conditioned media collected from COS-7 cells transiently transfected with full-length T $\beta$ RIII or sT $\beta$ RIII potently decreased TGF- $\beta$-induced invasion of MDAMB231 breast cancer cells through Matrigel (Figure 7, D and E).

As sT $\beta$ RIII mediated the effects of T $\beta$ RIII expression on breast cancer invasiveness in vitro and in vivo, we reasoned that T $\beta$ RIII would attenuate TGF- $\beta$ signaling in the MDA-MB231-T $\beta$ RIII cells in vitro and in the 4T1-T $\beta$ RIII tumors in vivo. To examine the effect of TRRIII expression on activation of the Smad pathway in response to TGF- $\beta$ stimulation, MDA-MB231-T $\beta$ RIII and MDA-MB231Neo breast cancer cells were treated with TGF- $\beta$, and phosphorylation levels of Smad2 were quantified. As shown in Figure 8A, T $\beta$ RIII expression in the MDA-MB231 cells resulted in reduced TGF- $\beta$-stimulated Smad2 phosphorylation compared with the MDA-MB231-Neo cells. In addition, TGF- $\beta 1$-mediated activation of TGF- $\beta 1$-responsive, Smad-dependent promoter pE2.1 was also reduced in the MDA-MB231-T $\beta$ RIII cells (Figure 8B). Consistent with this in vitro result, immunohistochemistry of the mouse mammary tumors revealed decreased frequency and intensity of phosphorylated Smad2 nuclear staining in the 4T1-T $\beta$ RIII tumors compared with the 4T1-Neo tumors (Figure 8C). Further support for a significant role for sT $\beta$ RIII in mediating the effects of T $\beta R I I I$ was provided by the decreased angiogenesis demonstrated in the 4T1-T $\beta$ RIII tumors in vivo (Figure 6B), as sT $\beta$ RIII has been demonstrated to decrease angiogenesis in vivo $(28,29)$.

sT $\beta$ RIII is produced from cells and tissues from 7 different mammalian species, including humans (30,31), and has also been detected in serum (30) and human milk (32). In addition, the expression of sT $\beta$ RIII has been demonstrated to closely correlate with the cell surface expression of T $\beta$ RIII (30), suggesting that it is released constitutively. To support a physiological role for sT $\beta$ RIII in mediating the effects of T $\beta$ RIII on breast cancer invasiveness, we examined expression of sT $\beta$ RIII in a panel of human breast epithelial and breast cancer cell lines. sT $\beta$ RIII was expressed in all human breast cell lines tested, including the human mammary epithelial cell line MCF-10A and the human breast cancer cell lines MCF-7, T47D, and MDA-MB231 (Supplemental Figure 3A). As previously reported, the level of sT $\beta$ RIII usually correlated with cell surface expression of T $\beta$ RIII. Finally, we examined expression of sT $\beta$ RIII in plasma from normal human volunteers as well as from patients with breast cancer. While we detected expression of sTRRIII (a het- 

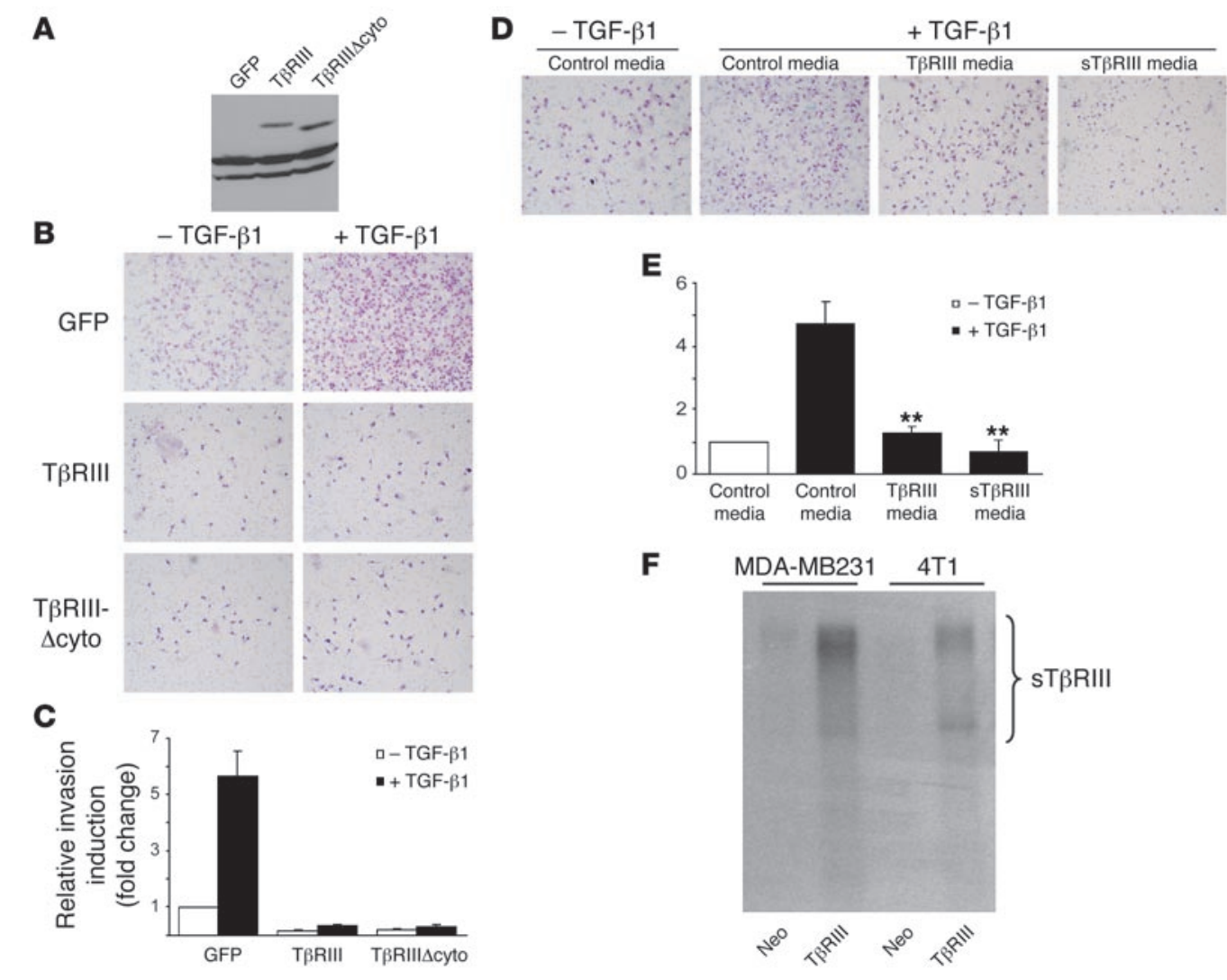

\section{Figure 7}

Restoration of T $\beta$ RIII expression inhibits Matrigel invasiveness of MDA-MB231 breast cancer cells. (A) MDA-MB231 cells were infected with equivalent amounts of adenoviral constructs carrying GFP, HA-tagged T $\beta$ RIII, and a T $\beta$ RIII mutant lacking the entire cytoplasmic domain (T $\beta$ RIII $\Delta$ cyto). Expression of the transgenes was confirmed by Western blotting of cell lysate using anti-HA antibody. (B and C) Matrigel invasion assay. Adenovirally infected MDA-MB231 cells ( 75,000 cells) were seeded in a Matrigel-coated upper chamber and treated with TGF- $\beta 1$ ( 15 pM) 2 hours later. Cell invasion through the Matrigel after 24 hours' incubation was detected by H\&E staining and quantitated. (D and E) Matrigel invasion assay was performed after resuspending MDA-MB231 cells in the conditioned media collected from pcDNA3.1-Neo-, T $\beta$ RIII-, and sT $\beta$ RIII-transfected COS-7 cells. Data are mean \pm SEM, $n=3$ in triplicate. ${ }^{* \star} P<0.01$. (F) Detection of sT $\beta$ RIII in media of MDA-MB231-T $\beta R I I I$ and 4 T1-T $\beta$ RIII cells by [125I]TGF- $\beta 1$-binding crosslinking followed by immunoprecipitation.

erogeneous product from approximately $65-250 \mathrm{kDa}$ ) in plasma in all (5 of 5) of the normal human volunteers, we did not detect sT $\beta$ RIII in the plasma of any breast cancer patients ( 0 of 13 ; Supplemental Figure 3B). Taken together, these data support a model in which ectodomain shedding of T $\beta$ RIII produces sT $\beta$ RIII, which then functions to attenuate TGF- $\beta$-mediated invasiveness of breast cancer cells and tumor-induced angiogenesis in vitro and in vivo.

Decreased T $\beta$ RIII expression correlates with decreased recurrence-free survival in breast cancer patients. As decreased T $\beta$ RIII expression is frequently observed in human breast cancers and restoring T $\beta$ RIII expression decreased invasiveness and metastasis in vivo, we explored whether T $\beta$ RIII expression could be a useful prognostic marker for breast cancer patients. We examined publicly available microarray data sets in which both T $\beta$ RIII expression and recurrence-free survival data were available (33-36). We set T $\beta$ RIII expression as a dichotomous variable, with high expression as above the mean and low expression as below the mean. In the largest data set (that of Wang et al., ref. 36), composed of 286 patients with lymph nodenegative breast cancers, low expression of T $\beta$ RIII was significantly associated with a decrease in recurrence-free survival (Figure 9; $P=0.043$ ), with recurrence defined as a distant metastatic event.
The hazard ratio (HR) for recurrence based on T $\beta$ RIII expression (HR, 1.569) was higher than that for ER status (HR, 1.18) or for Her2/Neu status (HR, 1.06) (37). In addition, we examined whether the predictive value of T $\beta$ RIII was independent of other known prognostic factors. As all samples in the Wang et al. data set (36) came from lymph node-negative patients, we analyzed the only other available prognostic factor within the data set, ER status. A Pearson correlation coefficient of -0.08 ( $95 \%$ confidence interval, -0.19 to 0.036 ) supported little correlation between T $\beta$ RIII expression and ER status, although the data set was not large enough to power the analysis $(P=0.177)$. In 3 other completely independent data sets (Sorlie et al., ref. 34, containing 74 locally advanced ERpositive and -negative primary breast cancers; van't Veer et al., ref. 33, containing 97 ER-positive and -negative lymph node-negative breast cancers; and Ma et al., ref. 35, containing 60 hormone receptor-positive breast cancers), there was a trend toward decreased recurrence-free survival associating with low T $\beta$ RIII expression, although in each case the number of patients was not large enough to reach statistical significance (data not shown). Taken together, these data suggest that T $\beta$ RIII expression is predictive of recurrence-free survival in breast cancer patients. 


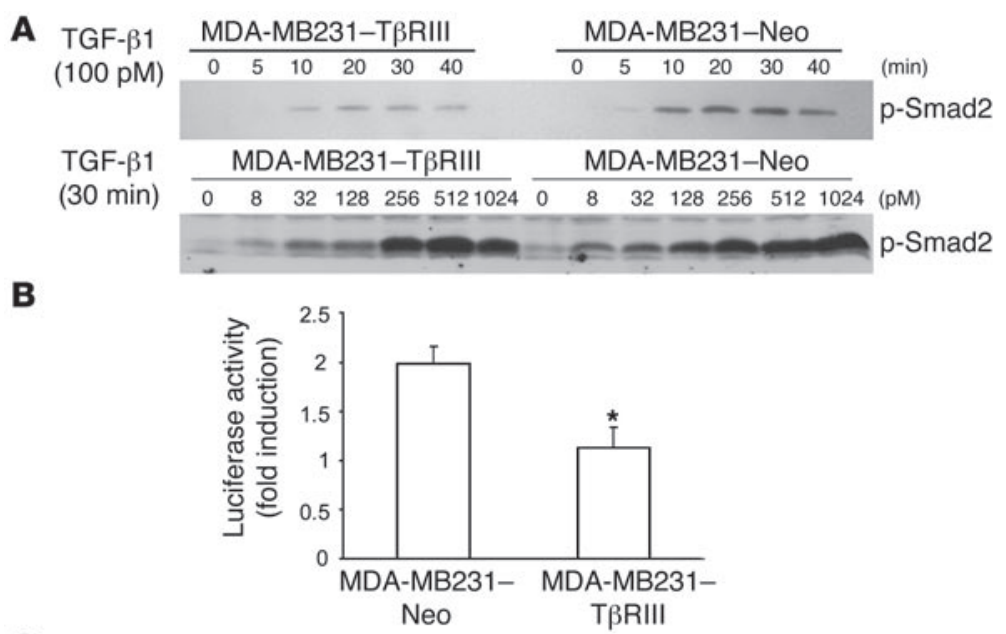

\section{Figure 8}

T $\beta$ RIII attenuates Smad2 phosphorylation in vitro and in vivo. (A) T $\beta$ RIII-overexpressing and control MDA-MB231 cells were treated with TGF- $\beta 1$ under the indicated conditions, and cell lysates were analyzed with a phosphoSmad2 ( $p$-Smad2) antibody. (B) Cells were transfected with $p E 2.1$ and $p S V \beta$ vector. Luciferase activity was determined after 24 hours of TGF- $\beta 1$ treatment $(100 \mathrm{pM})$ and is expressed as the fold induction over no TGF- $\beta$ treatment after adjusting for $\beta$-galactosidase expression. This assay was performed in triplicate at least 3 times. ${ }^{*} P<0.05$. (C) Phosphorylated Smad2 immunostaining of tissue sections from 4T1-Neo and 4T1-T $\beta$ RIII primary tumors. Representative results are shown. Note the significant decrease in staining intensity in the 4T1-T $\beta$ RIII tumor. Original magnification, $\times 40$.

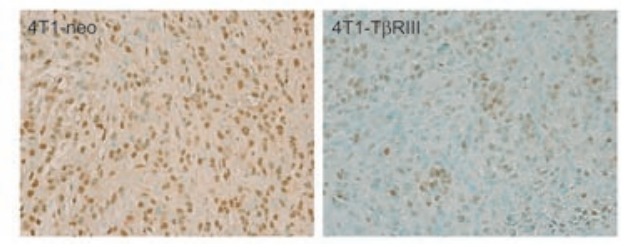

\section{Discussion}

Breast cancer is the leading cause of cancer death in women in the world, with most breast cancer morbidity and mortality resulting from metastatic disease (38). Although the TGF- $\beta$ signaling pathway has an important role in mammary carcinogenesis, the major components of the pathway, including the signaling receptors, T $\beta$ RII and $T \beta R I$, and the predominant signaling pathway downstream of these receptors, Smad2, Smad3, and Smad4, are usually intact in human breast cancers $(6,11)$. In the present study, we demonstrate that expression of the TGF- $\beta$ coreceptor T $\beta$ RIII was frequently decreased at the mRNA and protein levels in human breast cancer, with approximately $90 \%$ of specimens demonstrating decrease or loss at the mRNA level and approximately $70 \%$ demonstrating decrease or loss at the protein level. Thus, we believe loss of T $\beta$ RIII expression to be the most common alteration in the TGF- $\beta$ signaling pathway described in human breast cancer to date. We have further demonstrated that loss of T $\beta$ RIII expression was an early event, occurring initially in the preinvasive state, DCIS, with degree of loss correlating with breast cancer progression and corresponding to a decrease in patient survival. Mechanisms for decreased expression include $\mathrm{LOH}$ at the TGFBR3 gene locus and potential transcriptional downregulation of T $\beta$ RIII by elevated TGF- $\beta$ levels in the breast tumor microenvironment. Finally, we established a functional role for loss of T $\beta$ RIII expression, as restoring T $\beta$ RIII expression dramatically inhibited tumor invasiveness in vitro and tumor invasion, angiogenesis, and metastasis in vivo. Mechanistically, T $\beta$ RIII appeared to function by undergoing ectodomain shedding, with sT $\beta$ RIII antagonizing TGF- $\beta$ signaling and reducing invasiveness and angiogenesis in vivo. Taken together, these results support loss of T $\beta$ RIII expression as a frequent and important step in breast cancer progression, directly promoting breast cancer invasion and metastasis.

The dichotomous role of TGF- $\beta$ signaling in breast cancer development has been experimentally verified in several murine models. Specifically, blocking TGF- $\beta$ signaling in a series of human breast-derived cell lines representing different stages in breast cancer progression rendered premalignant cells tumorigenic, and low-grade tumorigenic cells more invasive, while making high-grade tumorigenic cells less metastatic (39). In addition, introduction of constitutively active T $\beta R$ I delayed oncogenic Neu-induced breast tumor onset but enhanced the frequency of lung metastasis in transgenic mice, whereas dominant-negative T $\beta$ RII enhanced Neu-induced tumor onset but decreased subsequent lung metastasis (40). Furthermore, inducing expression of active TGF- $\beta 1$ after primary breast tumor formation dramatically enhanced lung metastasis in a murine breast cancer model without a detectable effect on primary tumor size (41). Taken together, the results of these studies suggest that TGF- $\beta$ suppresses breast cancer progression in the early stages, but enhances tumor progression and metastasis in the later stages. Different explanations for this dichotomous function have been proposed, including TGF- $\beta$ exerting tumor-suppressing effects on epithelial-derived tumor cells and tumor-promoting effects on stromal cells (increased angiogenesis and immunosuppression, altered tumor cell-extracellular matrix interactions to enhance invasion and metastasis) (6). However,

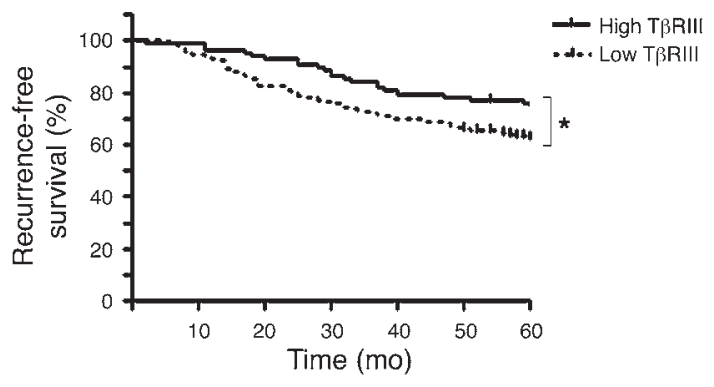

\section{Figure 9}

Low levels of T $\beta$ RIII predict decreased recurrence-free survival in women with breast cancer. Five-year recurrence-free survival for breast cancer with high or low T $\beta$ RIII expression was analyzed based on a microarray data set containing 286 patients. ${ }^{*} P<0.05$. 
emerging evidence suggests that TGF- $\beta$ may exert its dichotomous effects during carcinogenesis at least in part through biphasic effects on the epithelial derived cancer cells themselves, as the cells alter their molecular profiles to differentially respond to TGF- $\beta$ (6). Thus, even though resistant to the tumor suppressor effects of TGF- $\beta$ during tumorigenesis (growth inhibition, apoptosis, and differentiation), the cancer cells may respond to TGF- $\beta$ with increased motility and invasiveness. Based on the present findings, we propose that loss of $T \beta R I I I$ expression may be a mechanism for this differential response to TGF- $\beta$ during mammary carcinogenesis.

How might loss of T $\beta$ RIII expression alter cellular responses to TGF- $\beta$ during mammary carcinogenesis? Although T $\beta$ RIII was the first TGF- $\beta$ receptor cloned, as it has a short cytoplasmic domain with no intrinsic kinase activity, its role in TGF- $\beta$ signaling has not been well characterized. T $\beta$ RIII has classically been thought to act as a TGF- $\beta$ coreceptor, concentrating ligand on the cell surface and enhancing ligand binding to the signaling TGF- $\beta$ receptor T $\beta$ RII (42). However, emerging evidence supports a more substantial role for T $\beta$ RII in regulating and mediating TGF- $\beta$ signaling. T $\beta$ RIII has essential roles in chick (12) and murine development, with the T $\beta$ RIII knockout mouse having an embryonic lethal phenotype (13). In addition, we have previously established that regulating T $\beta$ RIII expression is sufficient to alter TGF- $\beta$ signaling (26), that the short cytoplasmic domain of T $\beta$ RIII is phosphorylated by T $\beta$ RII (14) and interacts with the PDZ domain-containing protein GIPC to stabilize T $\beta$ RIII expression on the cell surface and increasing TGF- $\beta$ signaling (26) as well as with the scaffolding protein $\beta$-arrestin 2 to mediate internalization of T $\beta$ RIII and T $\beta$ RII and downregulation of TGF- $\beta$ signaling (15). In addition, T $\beta$ RIII undergoes ectodomain shedding that releases the soluble extracellular domain (sT $\beta R I I I)$, which has been demonstrated to effectively neutralize TGF- $\beta$ and antagonize autocrine TGF- $\beta$ signaling. In breast cancer models, expressing sT $\beta$ RIII has been demonstrated to decrease tumorigenicity and spontaneous lung metastasis in immunocompromised mice through effects on both the tumor cells (decreasing cell growth and increasing apoptosis) (43) and the stroma (decreasing angiogenesis) $(28,29)$. While our results in the immunocompetent $4 \mathrm{~T} 1$ model confirm the effects of T $\beta$ RIII on angiogenesis, we found no significant effect of T $\beta$ RIII expression on cellular proliferation or apoptosis in either primary tumor or distant tumor metastastic lesions in vivo. Instead, in addition to decreased angiogenesis, the major effect of T $\beta$ RIII in vitro and in vivo was to decrease cellular invasiveness, with this effect mediated at least in part through the production of sT $\beta$ RIII. Therefore, we propose a model in which loss of T $\beta$ RIII expression results in alterations in TGF- $\beta$ responsiveness in both a cell-autonomous fashion (resulting in relative resistance of breast cancer cells to TGF- $\beta$ ) and a non-cell-autonomous fashion (by decreasing production of sT $\beta$ RIII), effectively increasing TGF- $\beta$ signaling in both the cancer cells and the stromal elements. Our in vitro and in vivo results demonstrating decreased Smad2 phosphorylation and decreased TGF- $\beta$ responsiveness in the presence of T $\beta$ RIII suggest that non-cell-autonomous regulation by sT $\beta$ RIII may have a dominant role in both tumor and stromal compartments. The contribution of T $\beta$ RIII and sT $\beta$ RIII on the balance of TGF- $\beta$ signaling and responsiveness in epithelial and stromal compartments remains an area of active investigation.

$\mathrm{T} \beta \mathrm{RIII}$ is located on chromosome $1 \mathrm{p} 32$, a region that frequently exhibits LOH in a wide variety of human cancers, including breast, colon, endometrial, gastric, kidney, lung, ovarian, and testicular cancer (20-22). For breast cancer, LOH at 1 p32 is associated with a poorer prognosis $(20,21)$. Previous studies have examined several potential tumor suppressor genes in this region, including mammary-derived growth inhibitor (44) and TP73 (45); however, expression and functional studies did not provide sufficient evidence supporting their role as tumor suppressor genes in breast cancer. In the present study, $\mathrm{LOH}$ analysis revealed allelic imbalance at the T $\beta$ RIII loci in $50 \%$ of the patients, with $\mathrm{LOH}$ correlating with loss of T $\beta$ RIII expression. The observed decrease in T $\beta$ RIII mRNA and protein expression could result from haploid insufficiency, as previously reported for TGF- $\beta 1$ (46), or from transcriptional downregulation or promoter hypermethylation of the remaining allele. The current data strongly support T $\beta$ RIII as a suppressor of breast cancer progression. T $\beta$ RIII has also been reported to be lost at an early stage in renal cell carcinogenesis (47). Whether T $\beta$ RIII functions as a suppressor of cancer progression in renal cell and other human cancers remains to be discerned.

Although breast cancer is thought to progress from a preinvasive state (DCIS) to invasive disease, we currently cannot determine which DCIS lesions are likely to remain indolent, and thus may be treated by local resection only, versus those DCIS lesions that will progress to invasive disease and/or recur, necessitating more aggressive treatment (i.e., postresection radiation, mastectomy, or adjuvant hormonal or chemotherapy). Clearly, understanding the molecular mechanisms by which DCIS becomes invasive and ultimately metastatic will allow identification of patients at low or high risk of recurrence and invasion/metastasis and guide these treatment options. In the present study, our data support loss of T $\beta$ RIII expression in DCIS as a common event potentially resulting in invasive and metastatic disease. Thus, as would be predicted, later-stage invasive cancers have a significantly higher frequency of T $\beta$ RIII loss, and lower T $\beta$ RIII expression correlates with a poorer prognosis for patients with invasive breast cancer. As this retrospective analysis was performed on patient tumor samples that were heterogeneous for both tumor and surrounding stromal tissue, we cannot be certain whether the loss in T $\beta$ RIII expression was in the tumor, stroma, or both, although our own IHC analysis of a large tissue array support that loss was primarily in tumor cells. Whether T $\beta$ RIII-negative DCIS lesions have a worse natural history and thus warrant more aggressive intervention than T $\beta$ RIII-positive DCIS lesions requires prospective validation.

\section{Methods}

TRRIII gene expression analysis on cDNA filter array. A filter array containing normalized cDNA from 50 breast cancers and corresponding normal tissues (Cancer Profiling Array; Clontech; Takara Bio Co.) was probed with [32P]-labeled cDNA probes for T $\beta R I I I$ following methods recommended by the manufacturer. In the 50 breast cancer samples, 33 were ductal carcinoma, 10 were lobular carcinoma, and 2 were tubular carcinoma; the remaining samples consisted of 1 each of mixed lobular/ductal carcinoma, medullary carcinoma, mucinous adenocarcinoma, fibrosarcoma, and DCIS. The T $\beta$ RIII cDNA probe was PCR amplified using the forward primer GTAGTGGGTTGGCCAGATGGT and reverse primer CTGCTGTCTCCCCTGTGTG. Purified PCR products $(25 \mathrm{ng})$ were labeled by random primed DNA labeling using $\left[\alpha^{-32} \mathrm{P}\right] \mathrm{dCTP}$ following the manufacturer's protocol (Roche Diagnostics). Labeled cDNA probe was purified on a BD CHROMA SPIN+STE-100 column (BD Biosciences - Clontech). Images were acquired using a phosphorimager, and subsequent data analysis was performed using NIH ImageJ software (http://rsb.info.nih.gov/ij/). A normal/tumor ratio of 2 or higher was considered to be significant.

Breast cancer tissue array. A polyclonal antibody recognizing T $\beta$ RIII protein was custom made by immunizing rabbits with a GST-fusion protein 
of the entire cytoplasmic domain of human T $\beta$ RIII. The IgG fraction of pre-immune and immune rabbit serum was collected using ImmunoPure IgG Purification kit (Pierce Biotechnology), and the specificity of the antibody was established by comparing staining of the breast cancer tissue arrays (Cooperative Breast Cancer Tissue Resource; National Cancer Institute) with preimmune serum and with the immune serum under identical conditions, by the specific pattern of staining of cells known to express T $\beta$ RIII (breast epithelial cells) and lack of staining of cells known not to express T $\beta$ RIII (lymphocytes), and by Western blot using protein extract from cell lines overexpressing human T $\beta$ RIII. Breast cancer tissue arrays (Cooperative Breast Cancer Tissue Resource) were deparaffinized, rehydrated, treated with $3 \%$ hydrogen peroxide, and then blocked with $10 \%$ goat serum. The arrays were incubated with anti-T $\beta$ RIII antibody overnight at $4^{\circ} \mathrm{C}$, washed in PBS, and further incubated with HRPconjugated anti-rabbit IgG (Vector Laboratory). Counterstaining was performed using hematoxylin. As a negative control, duplicate sections were immunostained with IgG purified from prebleed rabbit serum. The immunoreactivity for T $\beta$ RIII in breast epithelial and breast cancer cells was relatively uniform within a specimen and was thus semiquantitatively scored by staining intensity in a blinded manner with $0-1$ defined as no or weak staining, 2-3 as moderate staining, and 4-5 as intense staining. Standards for each staining score were used to maintain consistent scoring across specimens.

$\mathrm{LOH}$ and sequence analysis. Genomic DNA extracted from human breast cancer specimens and matching normal peripheral lymphocytes was kindly provided by the Breast Cancer Tissue Repository at Duke University. Microsatellite markers D1S1588, D1S188, D1S2804, and D1S435 were used in PCR reactions in which the forward primer was synthesized with a 5 fluorescent tag (Integrated DNA Technologies). PCR products were visualized using an ABI PRISM 3100 Genetic Analyzer (Applied Biosystems), and data were analyzed using GeneScan software (version 3.1; Applied Biosystems). $\mathrm{LOH}$ was determined as at least a $50 \%$ reduction in the relative intensity of one allele compared with the normal control. All samples positive for $\mathrm{LOH}$ were independently analyzed twice. PCR products were also analyzed on $3 \%$ MetaPhor agarose gels (Cambrex). For sequence analysis, 16 coding exons of T $\beta$ RIII were PCR amplified from the same DNA samples and subjected to sequence analysis (Supplemental Table 1).

Real-time PCR. Cells were treated with 100 pM TGF- $\beta 1$ for the times indicated in Figures 3 and 8 and the Figure 7 legend. Total RNA was isolated using RNeasy Mini Kit (QIAGEN), and first-strand cDNA was synthesized by M-MLV reverse transcriptase (Invitrogen). Quantitative real-time PCR was performed in the presence of iQ SYBR Green Supermix (Bio-Rad) on a Bio-Rad iCycler. Primer sequences are provided in Supplemental Table 2. Relative levels were calculated using the comparative threshold cycle method, with data normalized to GAPDH and expressed relative to untreated controls. All experiments were carried out in triplicate.

In vivo tumorigenicity and metastasis. Animal procedures were approved by the Institutional Animal Care and Use Committee of Duke University. The 4T1-Luc cell line stably expressing firefly luciferase gene under the selection of puromycin was generously provided by M.W. Dewhirst (Duke University Medical Center). These cells were further transfected with HA-tagged rat T $\beta$ RIII under the selection of neomycin and expression confirmed by ${ }^{[125}$ I]TGF- $\beta 1$ binding and crosslinking. Cells were implanted (50,000 cells/mouse) into the right-side axillary mammary gland of 7-week-old virgin, female BALB/c mice (Charles River Laboratories). Starting from day 10 after the implantation, growth of the tumors was measured with a caliber in 2 dimensions on alternate days and expressed as $\left(\right.$ length $\times$ width $\left.^{2}\right) \times 0.5$. On day 20 after implantation, surgical resection of the primary tumors was performed under sterile conditions. Four days after the surgery, tumor metastasis was recorded by bio- luminescence imaging of the mice every 3 days for 19 days. Briefly, mice were intraperitoneally injected with D-luciferin (Xenogen) at $150 \mu \mathrm{g} / \mathrm{g}$. Fifteen minutes following the injection, bioluminescence images were acquired using a IVIS camera (Xenogen). Bioluminescence for ROI was defined automatically, and data were expressed as photon flux (photons $/ \mathrm{s} / \mathrm{cm}^{2} /$ steradian). Background photon flux was defined from a ROI drawn over a mouse that was not given luciferin. At the end of the study mice were sacrificed, and sites of metastasis were determined by visual inspection. Interested organs were harvested for further IHC analysis and RNA and protein extraction. We used 4T1-Luc cells, stably transfected with the vector pcDNA3.1-Neo, in parallel as controls.

TUNEL, PCNA, CD31, and phosphorylated Smad2 immunostaining. TUNEL and PCNA immunostainings were performed on paraffin-embedded tissue sections according to the manufacturer's instructions (TUNEL, In Situ Cell Death Detection kit, POD; Roche Diagnostics; PCNA, Santa Cruz Biotechnology Inc.). CD31 immunostaining was performed on frozen tissue sections as specified by the manufacturer (Cell Signaling Technology). For phosphorylated Smad2 staining, antigen retrieval was carried out by boiling slides in $10 \mathrm{mM}$ citrate buffer ( $\mathrm{pH}$ 6.0) for 5 minutes in a microwave after blocking in $3 \%$ hydrogen peroxide.

Matrigel invasion assay. We seeded 75,000 cells in the Matrigel-coated upper chamber (BD Biosciences) of a 24-well transwell. Media containing $10 \%$ FBS was placed in the lower chamber as a chemoattractant. After 2 hours' incubation, 15 pM TGF- $\beta 1$ was added into the designated upper chambers. Twenty-four hours later, the cells on the upper surface of the filter were removed by gently scrubbing with a cotton swab. The cells that migrated to the underside of the filter were fixed and stained with H\&E. Each filter was removed and examined microscopically, and 3 random images were acquired. Cells present in each image were counted. In some assays, conditioned serum-free medium collected from COS-7 cells transiently transfected with empty vector, full-length T $\beta$ RIII, or sT $\beta$ RIII construct was used to resuspend cells to be seeded in the upper chambers. These assays were performed in triplicate at least 3 times.

TGF- $\beta$ binding and crosslinking assay. We incubated $100 \mathrm{pM}$ [125I]TGF- $\beta 1$ with $500 \mu \mathrm{l}$ of the cell medium in the presence of protease inhibitors for 3 hours at $4^{\circ} \mathrm{C}$. The $\left[{ }^{125} \mathrm{I}\right] \mathrm{TGF}-\beta 1-\mathrm{sT} \beta \mathrm{RIII}$ complex was then crosslinked with $0.5 \mathrm{mg} / \mathrm{ml}$ disuccinimidyl suberate and immunoprecipitated with a polyclonal antibody recognizing the extracellular domain of T $\beta$ RIII (R\&D Systems). The final complex was visualized after SDS-PAGE and autoradiography.

Transcription reporter luciferase assays. Cells were transfected with a pE2.1 vector that contains the luciferase gene under the regulation of a promoter based on the TGF- $\beta$-inducible promoter PAI- 1 and the PSV $\beta$ vector encoding $\beta$-galactosidase as a control for transfection efficiency. After 24 hours, the cells were treated with TGF- $\beta 1$ (100 pM) for an additional 24-hour period. The cells were lysed in luciferase lysis buffer (Promega). The luciferase activity was read after the addition of luciferin (Promega) using an automated luminometer. The luciferase activity was expressed as the fold induction over no TGF- $\beta$ treatment after adjusting for $\beta$-galactosidase expression.

$\left[{ }^{3} \mathrm{H}\right]$ Thymidine incorporation assay. Cells growing in 24-well plates were treated with $0-200$ pm TGF- $\beta 1$ for 48 hours when they reached $80 \%$ confluency and then incubated with $10 \mu \mathrm{Ci}$ of $\left[{ }^{3} \mathrm{H}\right]$ thymidine (Amersham Biosciences) for an additional 4 hours. Cells were washed with PBS and 5\% trichloroacetic acid before being harvested with $0.1 \mathrm{~N} \mathrm{NaOH}$. The amount of incorporated $\left[{ }^{3} \mathrm{H}\right]$ thymidine was determined by scintillation counting. Growth inhibition was calculated as the ratio of radioactivity with TGF- $\beta 1$ treatment to radioactivity without TGF- $\beta 1$ treatment.

Statistics. Statistical analysis was performed using the 2-tailed Student's $t$ test unless otherwise indicated. All data are presented as mean \pm SEM. $P$ values less than 0.05 were considered to be significant. 


\section{Acknowledgments}

Tissue microarray slides were provided by the Cooperative Breast Cancer Tissue Resource, which is funded by the National Cancer Institute. We thank M.W. Dewhirst for the 4T1-Luc cell line. We thank N. Glover and J. Fuller for technical assistance. These studies were supported by National Institutes of Health/National Cancer Institute grant R01-CA106307 (to G.C. Blobe), by a postdoctoral fellowship from the Susan G. Komen Breast Cancer Foundation (to M. Dong), and by predoctoral fellowships from the Department of Defense Breast Cancer Research Program (to K.C. Kirkbride and J.D. Lee).
Received for publication June 5, 2006, and accepted in revised form October 10, 2006.

Address correspondence to: Gerard C. Blobe, 221B MSRB Research Drive, Box 2631 DUMC, Durham, North Carolina 27710, USA. Phone: (919) 668-1352; Fax: (919) 668-2458; E-mail: blobe001@mc.duke.edu.

Tam How, Kellye C. Kirkbride, Kelly J. Gordon, Jason D. Lee, and Nadine Hempel contributed equally to this work.
1. Massague, J. 1998. TGF-beta signal transduction. Annu. Rev. Biochem. 67:753-791.

2. Jhappan, C., et al. 1993. Targeting expression of a transforming growth factor beta 1 transgene to the pregnant mammary gland inhibits alveolar development and lactation. EMBO J. 12:1835-1845.

3. Pierce, D.F., Jr., et al. 1993. Inhibition of mammary duct development but not alveolar outgrowth during pregnancy in transgenic mice expressing active TGF-beta 1. Genes Dev. 7:2308-2317.

4. Pierce, D.F., Jr., et al. 1995. Mammary tumor suppression by transforming growth factor beta 1 transgene expression. Proc. Natl. Acad. Sci. U. S. A. 92:4254-4258.

5. Decensi, A., et al. 1998. Correlation between plasma transforming growth factor-beta 1 and second primary breast cancer in a chemoprevention trial. Eur. J. Cancer. 34:999-1003.

6. Elliott, R.L., and Blobe, G.C. 2005. Role of transforming growth factor Beta in human cancer. J. Clin. Oncol. 23:2078-2093.

7. Walker, R.A., and Dearing, S.J. 1992. Transforming growth factor beta 1 in ductal carcinoma in situ and invasive carcinomas of the breast. Eur. J. Cancer. 28:641-644.

8. Dalal, B.I., Keown, P.A., and Greenberg, A.H. 1993. Immunocytochemical localization of secreted transforming growth factor-beta 1 to the advancing edges of primary tumors and to lymph node metastases of human mammary carcinoma. Am.J. Pathol. 143:381-389.

9. Gorsch, S.M., Memoli, V.A., Stukel, T.A., Gold, L.I., and Arrick, B.A. 1992. Immunohistochemical staining for transforming growth factor beta 1 associates with disease progression in human breast cancer. Cancer Res. 52:6949-6952.

10. Ghellal, A., et al. 2000. Prognostic significance of TGF beta 1 and TGF beta 3 in human breast carcinoma. Anticancer Res. 20:4413-4418.

11. Xie, W., et al. 2002. Alterations of Smad signaling in human breast carcinoma are associated with poor outcome: a tissue microarray study. Cancer Res. 62:497-505.

12. Brown, C.B., Boyer, A.S., Runyan, R.B., and Barnett, J.V. 1999. Requirement of type III TGF-beta receptor for endocardial cell transformation in the heart. Science. 283:2080-2082.

13. Stenvers, K.L., et al. 2003. Heart and liver defects and reduced transforming growth factor beta2 sensitivity in transforming growth factor beta type III receptor-deficient embryos. Mol. Cell. Biol. 23:4371-4385.

14. Blobe, G.C., et al. 2001. Functional roles for the cytoplasmic domain of the type III transforming growth factor beta receptor in regulating transforming growth factor beta signaling. J. Biol. Chem. 276:24627-24637.

15. Chen, W., et al. 2003. Beta-arrestin 2 mediates endocytosis of type III TGF-beta receptor and down-regulation of its signaling. Science. 301:1394-1397.

16. Chen, C., Wang, X.F., and Sun, L. 1997. Expression of transforming growth factor beta (TGFbeta) type III receptor restores autocrine TGFbeta1 activity in human breast cancer MCF-7 cells. J. Biol. Chem. 272:12862-12867.
17. Sun, L., and Chen, C. 1997. Expression of transforming growth factor beta type III receptor suppresses tumorigenicity of human breast cancer MDA-MB231 cells. J. Biol. Chem. 272:25367-25372.

18. Parsons, R., et al. 1995. Microsatellite instability and mutations of the transforming growth factor beta type II receptor gene in colorectal cancer. Cancer Res. 55:5548-5550.

19. Hahn, S.A., et al. 1996. DPC4, a candidate tumor suppressor gene at human chromosome 18q21.1. Science. 271:350-353.

20. Ragnarsson, G., et al. 1999. Loss of heterozygosity at chromosome $1 \mathrm{p}$ in different solid human tumours: association with survival. Br. J. Cancer. 79:1468-1474.

21. Borg, A., Zhang, Q.X., Olsson, H., and Wenngren, E. 1992. Chromosome 1 alterations in breast cancer: allelic loss on $1 \mathrm{p}$ and $1 \mathrm{q}$ is related to lymphogenic metastases and poor prognosis. Genes Chromosomes Cancer. 5:311-320.

22. Bieche, I., Khodja, A., and Lidereau, R. 1999. Deletion mapping of chromosomal region 1p32-pter in primary breast cancer. Genes Chromosomes Cancer. 24:255-263.

23. Ji, C., Chen, Y., McCarthy, T.L., and Centrella, M. 1999. Cloning the promoter for transforming growth factor-beta type III receptor. Basal and conditional expression in fetal rat osteoblasts. J. Biol. Chem. 274:30487-30494.

24. Pulaski, B.A., and Ostrand-Rosenberg, S. 1998. Reduction of established spontaneous mammary carcinoma metastases following immunotherapy with major histocompatibility complex class II and B7.1 cell-based tumor vaccines. Cancer Res. 58:1486-1493.

25. Heppner, G.H., Miller, F.R., and Shekhar, P.M. 2000. Nontransgenic models of breast cancer. Breast Cancer Res. 2:331-334.

26. Blobe, G.C., Liu, X., Fang, S.J., How, T., and Lodish, H.F. 2001. A novel mechanism for regulating transforming growth factor beta (TGF-beta) signaling. Functional modulation of type III TGF-beta receptor expression through interaction with the PDZ domain protein, GIPC. J. Biol. Chem. 276:39608-39617.

27. Lopez-Casillas, F., Payne, H.M., Andres, J.L., and Massague, J. 1994. Betaglycan can act as a dual modulator of TGF-beta access to signaling receptors: mapping of ligand binding and GAG attachment sites. J. Cell Biol. 124:557-568.

28. Bandyopadhyay, A., et al. 2002. Extracellular domain of TGFbeta type III receptor inhibits angiogenesis and tumor growth in human cancer cells. Oncogene. 21:3541-3551.

29. Bandyopadhyay, A., et al. 2002. Antitumor activity of a recombinant soluble betaglycan in human breast cancer xenograft. Cancer Res. 62:4690-4695.

30. Andres, J.L., Stanley, K., Cheifetz, S., and Massague, J. 1989. Membrane-anchored and soluble forms of betaglycan, a polymorphic proteoglycan that binds transforming growth factor-beta. J. Cell Biol. 109:3137-3145.

31. Philip, A., Hannah, R., and O'Connor-McCourt, M. 1999. Ectodomain cleavage and shedding of the type III transforming growth factor-beta receptor in lung membranes effect of temperature, ligand binding and membrane solubilization. Eur. J. Biochem. 261:618-628.

32. Cheung, H.K., Mei, J., and Xu, R.J. 2003. Quantification of soluble betaglycan in porcine milk. Asia Pac. J. Clin. Nutr. 12(Suppl.):S61.

33. van't Veer, L.J., et al. 2002. Gene expression profiling predicts clinical outcome of breast cancer. Nature. 415:530-536.

34. Sorlie, T., et al. 2003. Repeated observation of breast tumor subtypes in independent gene expression data sets. Proc. Natl. Acad. Sci. U. S. A. 100:8418-8423.

35. Ma, X.J., et al. 2004. A two-gene expression ratio predicts clinical outcome in breast cancer patients treated with tamoxifen. Cancer Cell. 5:607-616.

36. Wang, Y., et al. 2005. Gene-expression profiles to predict distant metastasis of lymph-node-negative primary breast cancer. Lancet. 365:671-679.

37. Moody, S.E., et al. 2005. The transcriptional repressor Snail promotes mammary tumor recurrence. Cancer Cell. 8:197-209.

38. Kamangar, F., Dores, G.M., and Anderson, W.F. 2006. Patterns of cancer incidence, mortality, and prevalence across five continents: defining priorities to reduce cancer disparities in different geographic regions of the world. J. Clin. Oncol. 24:2137-2150.

39. Tang, B., et al. 2003. TGF- $\beta$ switches from tumor suppressor to prometastatic factor in a model of breast cancer progression. J. Clin. Invest. 112:1116-1124. doi:10.1172/JCI200318899.

40. Siegel, P.M., Shu, W., Cardiff, R.D., Muller, W.J., and Massague, J. 2003. Transforming growth factor beta signaling impairs Neu-induced mammary tumorigenesis while promoting pulmonary metastasis. Proc. Natl. Acad. Sci. U. S. A. 100:8430-8435.

41. Muraoka-Cook, R.S., et al. 2004. Conditional overexpression of active transforming growth factor beta 1 in vivo accelerates metastases of transgenic mammary tumors. Cancer Res. 64:9002-9011.

42. Lopez-Casillas, F., Wrana, J.L., and Massague, J. 1993. Betaglycan presents ligand to the TGF beta signaling receptor. Cell. 73:1435-1444.

43. Lei, X., Bandyopadhyay, A., Le, T., and Sun, L. 2002. Autocrine TGFbeta supports growth and survival of human breast cancer MDA-MB-231 cells. Oncogene. 21:7514-7523.

44. Huynh, H., Alpert, L., and Pollak, M. 1996. Silencing of the mammary-derived growth inhibitor (MDGI) gene in breast neoplasms is associated with epigenetic changes. Cancer Res. 56:4865-4870.

45. Ahomadegbe, J.C., et al. 2000. Loss of heterozygosity, allele silencing and decreased expression of $\mathrm{p} 73$ gene in breast cancers: prevalence of alterations in inflammatory breast cancers. Oncogene. 19:5413-5418.

46. Tang, B., et al. 1998. Transforming growth factorbeta 1 is a new form of tumor suppressor with true haploid insufficiency. Nat. Med. 4:802-807.

47. Copland, J.A., et al. 2003. Genomic profiling identifies alterations in TGFbeta signaling through loss of TGFbeta receptor expression in human renal cell carcinogenesis and progression. Oncogene. 22:8053-8062. 